\title{
A case of Malakoplakia Mimicking Prostatic Malignancy
}

\author{
Kshitija M Kale*, Anitha Padmanabhan, Prapti Acharya and Nitin M Gadgil
}

Department of Pathology, Lokmanya Tilak Municipal Medical College and Sion Hospital, Mumbai, India

\section{ABSTRACT}

Malakoplakia is a granulomatous inflammatory condition commonly involving the urogenital tract. Principally it involves the urinary bladder, prostate is a rare site. Clinical and radiological examination sometimes mimics malignancy. Histology remains confirmatory modality for the diagnosis. Hereby we report a case of prostatic malakoplakia in an elderly male who presented with lower urinary tract symptoms in the past fifteen days. Urine routine microscopy showed pus cells; culture report was positive for E. coli. Serum Prostate Specific antigen (PSA) was $4.6 \mathrm{ug} / \mathrm{ml}$. Magnetic Resonance Imaging (MRI) showed a bulky prostate. A 12-core biopsy was done to rule out malignancy and the histopathology showed benign prostatic glands and the stroma with a predominant population of epithelioid histiocytes and the characteristic Michealis-Gutmann bodies. After a course of antibiotic Nitrofurantoin, transrectal ultrasound was repeated which showed reduction in the prostatic volume. Subsequently Trans-Urethral Resection of Prostate (TURP) was done to relieve the persistent lower urinary tract symptoms. Histology showed similar features. Special stains - Von kossa and Prussian Blue highlighted the Michealis-Gutmann bodies.

\section{Keywords: Malakoplakia, Prostate, Malignancy Mimic, Michealis-Gutmann Bodies}

\section{Introduction}

Malakoplakia is a granulomatous inflammatory condition involving all the systems of the body, urogenital tract being the commonest. Principally it involves the urinary bladder, prostate is a rare site. The etiopathogenesis stems from an infection by the bacteria Escherichia Coli (E. coli) and a defective immune response to it. Histologically it is characterised by foamy histiocytes, inflammatory cells and Michaelis-Gutmann (MG) bodies. Clinical and radiological examination sometimes mimics malignancy. Histology remains confirmatory modality for the diagnosis.

\section{Case Report:}

A 75-year elderly male presented with lower urinary tract symptoms in the past 15 days. Digital rectal examination (DRE) revealed a firm to hard enlarged prostate; grade 2 prostatomegaly. Urine routine microscopy showed pus cells ( 8 to 10 per hpf) and culture report was positive for E. coli organisms ( $>10 \square \mathrm{CFU} / \mathrm{ml})$. Serum PSA was 4.6ug/ $\mathrm{ml}$. Transrectal ultrasound revealed prostate volume 95 cc. MRI showed bulky prostate, diffuse T2 hypo intensity and ill-defined T2 hypointense nodules. The presence of hypointense nodules on T2 imaging MRI pointed towards malignancy. Post contrast studies did not reveal any abnormal enhancement. (fig. 1). A 12-core biopsy was done to rule out malignancy which was strongly suspected on DRE and radiology imaging. Histological examination showed benign prostatic glands and the stroma with a predominant population of epithelioid histiocytes - large cells with clear abundant cytoplasm and the characteristic MG bodies which appeared round to oval, concentrically laminated and stained pink with Haematoxylin and Eosin. These bodies were found within the epithelioid histiocytes and in the interstitium. After a course of antibiotic Nitrofurantoin, transrectal ultrasound was repeated which showed reduction in the prostatic volume to $35 \mathrm{cc}$. Subsequently TURP was done to relieve the persistent lower urinary tract symptoms. Histology of TURP chips showed similar features (fig. 2 \& 3). Special stains Von kossa and Prussian Blue highlighted the MichaelisGutmann bodies(fig.4). our patient is now symptom free and the follow up is unremarkable.

\section{Discussion}

Urogenital tract is the most common site for malakoplakia. However, prostatic malakoplakia is rare with less than 50 cases reported in literature (1). The pathogenesis of malakoplakia lies in the impaired histiocytic response against bacteria, most commonly E. coli. The ultrastructural basis of this pathogenesis is related to defect in lysosomes and abnormality in microtubule assembly ${ }^{(2)}$. There is a higher incidence of these cases in individuals with impaired immunity, commonly Diabetes mellitus and HIV infection. There are occasional case reports of co-existing malakoplakia and prostatic adenocarcinoma (3). However, in these patients malakoplakia is presumed to be a complication of infection following TRUS biopsy. Majority of the cases reported in literature were thought 


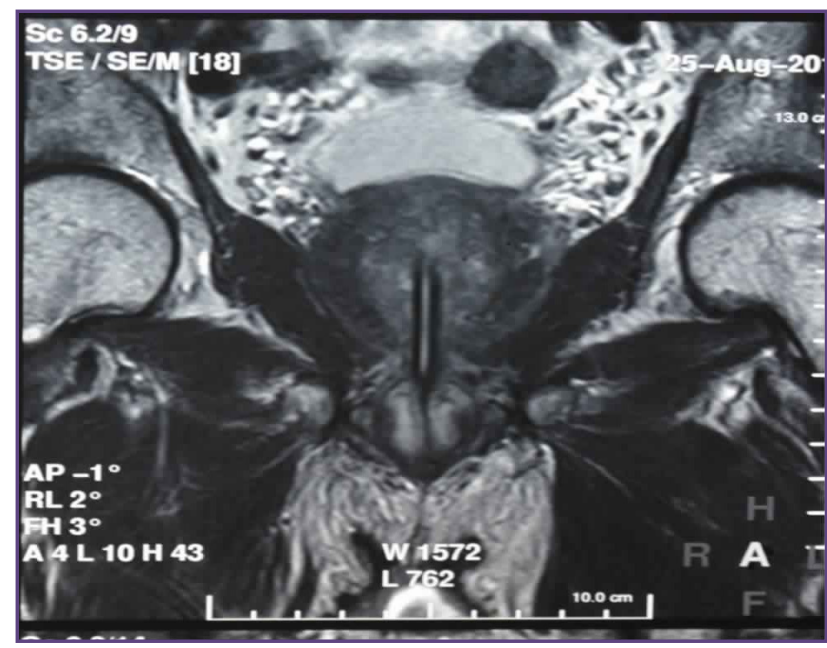

Fig. 1: T2 MRI: Bulky prostate, Diffuse T2 hypointense with ill defined hypointense nodules.

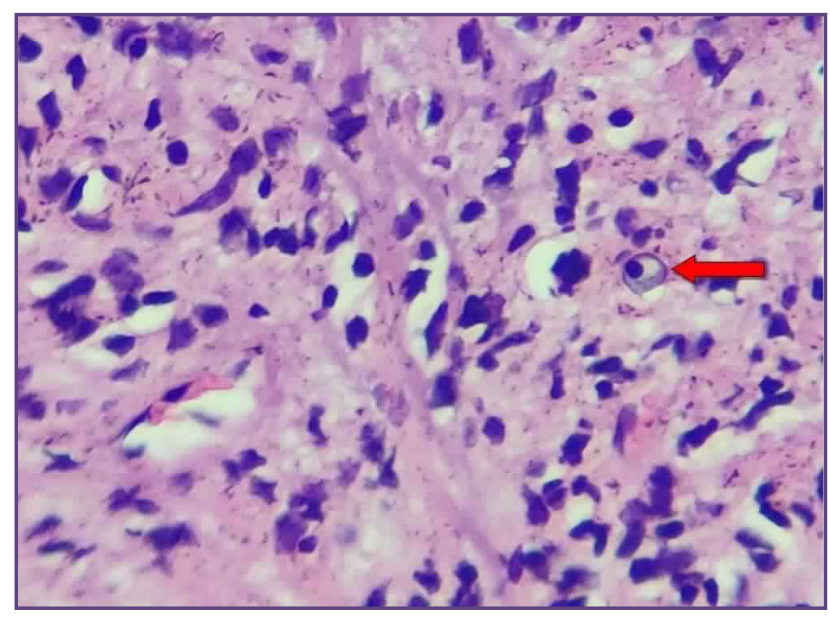

Fig. 3: 4oox, H \& E. Concenttric lamelleted structuresMichaelis-Gutmann bodies.

to be neoplastic on clinical and radiological examination as digital rectal examination revealed hard nodules and MRI showed bulky prostate with hypointense nodules ${ }^{(4)}$. However, the diagnosis was confirmed as malakoplakia on histology. In few patient's malakoplakia is diagnosed incidentally in TURP chips. Antibiotic therapy is the mainstay of treatment. Surgery may be required depending on the extent of the disease ${ }^{(5)}$. In our patient the prostatic volume reduced significantly with antibiotic course however the obstructive symptoms persisted. Hence TURP was performed. The patient is now symptom free.

\section{Conclusion}

Malakoplakia in prostate is rarely encountered in clinical practice. It is sometimes misdiagnosed as carcinoma during clinical and radiological examination. It can be easily treated with a course of antibiotics. Histopathological

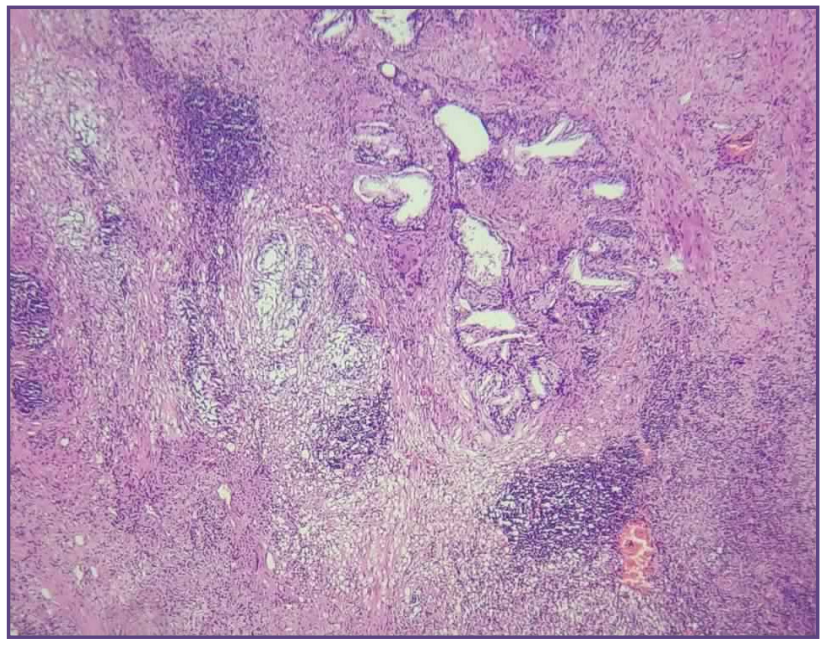

Fig. 2: 40x H \& E. Benign prostatic glands with areas of imflammatory cells, clear cells and epithelioid histiocytes.

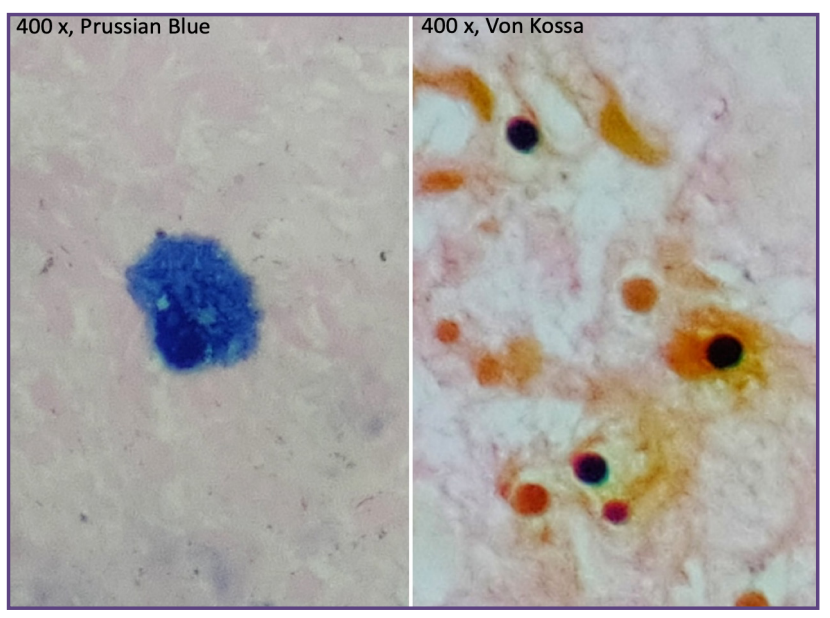

Fig. 4: Special stains: Highlights the Michaelis-Gutmann bodies.

examination remains the gold standard for confirmatory diagnosis of malakoplakia.

\section{Acknowledgements}

Dr. L P Naik, Professor and Head, Department of Pathology, LTMMC and LTMGH, Sion.

\section{Reference}

1. Matthew Ho, Jeremy Wu, Brian S, Alex K. Prostatic Malakoplakia: a case report with review of literature. Journal of Surgical Case Reports 2018; 3: 1-3.

2. Dasgupta P, Womack C, Turner AG, Blackford HN. Malakoplakia: von Hansemann's disease. BJU Int. 1999; 84(4): 464-9.

3. Medlicott S, Magi-Galluzzi C, Jimenez R, Trpkov K. Malakoplakia associated with prostatic adenocarcinoma: report of 4 cases and literature review. Ann diagn pathol 2016; $22: 33-7$. 
4. Dale R, Metcalfe M, Chang S, Jones E, Black P. Malakoplakia of the prostate masquerading as locally advanced prostate cancer on mpMRI. Can Urol Assoc J 2015; 9: 910-2.
5. Kahraman DS, Sayhan S, Diniz G, Ayaz D, Karadeniz T et al. A pit fall in transrectal prostate biopsy: Malakoplakia evaluation of two cases based on the literature review. Case Reports in Pathology 2014; 2014: 1-3.

*Corresponding author:

Dr. Kshitija Kale, A7-1-1, Millennium Towers, Sector-9, Sanpada, Navi Mumbai - 400705 INDIA.

Phone: +91 9892989692

Email: mrudulakm@gmail.com

Financial or other Competing Interests: None. 